\title{
Qualitative study of system-level factors related to genomic implementation
}

\author{
Alexis M. Zebrowski, MPH ${ }^{1,2}$, Darcy E. Ellis, MPH ${ }^{1}$, Frances K. Barg, PhD, MEd ${ }^{1,3}$, \\ Nina R. Sperber, PhD, MA ${ }^{4}$, Barbara A. Bernhardt, MS, $\mathrm{CGC}^{5}$, Joshua C. Denny, MD, MS ${ }^{6,7}$, \\ Paul R. Dexter, $\mathrm{MD}^{8}$, Geoffrey S. Ginsburg, MD, PhD ${ }^{9}$, Carol R. Horowitz, MD, MPH ${ }^{10}$, \\ Julie A. Johnson, PharmD ${ }^{11}$, Mia A. Levy, MD, PhD $^{12}$, Lori A. Orlando, MD, $\mathrm{MHS}^{9,13}$, \\ Toni I. Pollin, PhD, CGC ${ }^{14}$, Todd C. Skaar, PhD ${ }^{15}$ and Stephen E. Kimmel, MD, MSCE ${ }^{1,2,16}$
}

Purpose: Research on genomic medicine integration has focused on applications at the individual level, with less attention paid to implementation within clinical settings. Therefore, we conducted a qualitative study using the Consolidated Framework for Implementation Research (CFIR) to identify system-level factors that played a role in implementation of genomic medicine within Implementing GeNomics In PracTicE (IGNITE) Network projects.

Methods: Up to four study personnel, including principal investigators and study coordinators from each of six IGNITE projects, were interviewed using a semistructured interview guide that asked interviewees to describe study site(s), progress at each site, and factors facilitating or impeding project implementation. Interviews were coded following CFIR inner-setting constructs.

Results: Key barriers included (1) limitations in integrating genomic data and clinical decision support tools into electronic health records, (2) physician reluctance toward genomic research participation and clinical implementation due to a limited evidence base, (3) inadequate reimbursement for genomic medicine, (4) communication among and between investigators and clinicians, and (5) lack of clinical and leadership engagement.

Conclusion: Implementation of genomic medicine is hindered by several system-level barriers to both research and practice. Addressing these barriers may serve as important facilitators for studying and implementing genomics in practice.

Genetics in Medicine (2019) 21:1534-1540; https://doi.org/10.1038/s41436018-0378-9

Keywords: implementation; qualitative; genomics; electronic health record; clinical engagement

\section{INTRODUCTION}

Dramatic advances in genomic discoveries and science have led to repeated predictions that genomic medicine will revolutionize health-care delivery. ${ }^{1-3}$ The potential clinical applications of genomic advances are vast and include improved assessment of disease risk, personalized targeted therapies and risk reduction strategies, and the potential for avoiding adverse drug reactions. ${ }^{4-6}$ Several specific applications of genomic advances are being incorporated successfully into clinical care in some settings, including the use of tumor sequencing for selection of treatment based on the molecular characterization of the tumor, the use of genome or exome sequencing for the diagnosis of rare disorders, and preemptive genotyping to guide medication prescribing at the point of care. $^{4,6-9}$

Given the potential for such dramatic improvements in personal and public health, genomics deserves special consideration of how to successfully integrate advances into clinical care. Similar to the incorporation of most new technologies, clinical integration of genomic medicine has

\footnotetext{
${ }^{1}$ Department of Biostatistics, Epidemiology, and Informatics, University of Pennsylvania, Philadelphia, PA, USA; ${ }^{2}$ Leonard Davis Institute of Health Economics, University of Pennsylvania, Philadelphia, PA, USA; ${ }^{3}$ Department of Family Medicine and Community Health, University of Pennsylvania, Philadelphia, PA, USA; ${ }^{4}$ Center for Health Services Research in Primary Care, Durham Veterans Affairs Medical Center, Durham, NC, USA; ${ }^{5}$ Division of Translational Medicine and Human Genetics, Perelman School of Medicine, University of Pennsylvania, Philadelphia, PA, USA; ${ }^{6}$ Departments of Biomedical Informatics and Medicine, Vanderbilt University School of Medicine, Nashville, TN, USA; ${ }^{7}$ Division of General Internal Medicine, Department of Medicine, Vanderbilt University School of Medicine, Nashville, TN, USA; ${ }^{8}$ Department of Medicine, Indiana University School of Medicine, Indianapolis, IN, USA; ${ }^{9}$ Duke Center for Applied Genomics and Precision Medicine, Duke University Medical Center, Durham, NC, USA; ${ }^{10}$ Department of Population Health Science and Policy, Icahn School of Medicine at Mount Sinai, New York, NY, USA; ${ }^{11}$ Department of Pharmacotherapy and Translational Research and Center for Pharmacogenomics, University of Florida College of Pharmacy, Gainesville, FL, USA; ${ }^{12}$ Departments of Biomedical Informatics and Medicine, Division of Hematology and Oncology, Vanderbilt University School of Medicine, Nashville, TN, USA; ${ }^{13}$ Division of General Internal Medicine, Department of Medicine, Duke University Medical Center, Durham, NC, USA; ${ }^{14}$ University of Maryland School of Medicine, Baltimore, MD, USA; ${ }^{15}$ Division of Clinical Pharmacology, Indiana University School of Medicine, Indianapolis, IN, USA; ${ }^{16}$ Department of Medicine, Pearlman School of Medicine, University of Pennsylvania, Philadelphia, PA, USA. Correspondence:

Stephen E. Kimmel (stevek@pennmedicine.upenn.edu)
} 
been slow, even for applications with proven effectiveness. ${ }^{8,10}$ The lag has been attributed to multiple factors relevant to a variety of stakeholders, including limited evidence that genetic tests improve individual health outcomes and a lack of clinician education about genomic medicine. ${ }^{4,8,10-12}$ The lack of evidence of efficacy in most genomic medicine applications has been a major challenge, leading to recommendations for alternatives to randomized controlled trials, new models of comparative effectiveness research, and emphasis on the importance of patient utility and the ability of patients to interpret genomic test results to make decisions, take action, or better understand themselves and their health. ${ }^{13-15}$ However, barriers for the conduct of such research have been largely unexplored.

Although inadequate physician understanding of genetics has been acknowledged for decades, leading to calls for more education, ${ }^{16-18}$ physicians still are generally reluctant to incorporate genomics and genetic risk assessment into their clinical practice, and some lack confidence in their ability to relay results to their patients. ${ }^{19-22}$ Some are also skeptical of the impact genomic medicine would have on patient care and some require more professional education to better integrate it into standard patient care. ${ }^{19,22}$ Other barriers relate to concerns about the ethical, legal, and social implications of genetic testing and technologies, leading both patients and clinicians to be concerned about risks associated with their use. $^{23}$

To gather data on optimal practices for successful and sustained integration of genomic medicine into practice, the National Institutes of Health (NIH) National Human Genome Research Institute (NHGRI) funded six genomic medicine demonstration projects aimed at (1) linking existing genomic medicine efforts, (2) developing new collaborative projects in diverse settings and populations, (3) providing evidence to support the use of genomic information in clinical care, and (4) sharing best practices for genomic medicine implementation, diffusion, and sustainability. ${ }^{8,24}$ These clinical studies comprised the Implementing GeNomics In PracTicE (IGNITE) Network, and employed diverse genomic medicine interventions (e.g., genetic testing for tailoring drug prescribing, including CYP2C19 genotyping for clopidogrel and APOL1 testing for risk of end stage kidney disease in African Americans with hypertension, testing for monogenic diabetes, and evaluation of a family history tool for use by primary care providers) in a variety of clinical settings. ${ }^{24}$ These settings include primary care and community health clinics, inpatient units, and academic medical centers.

Much of the research on genomic medicine integration to date has focused on identifying and addressing barriers of single applications of genomic medicine at the level of the individuals directly involved with implementation, primarily patients and providers. ${ }^{8}$ Less attention has been paid to rigorously examining barriers to implementation from an organizational, system-level perspective across a broad range of clinical practice settings. Additionally, understanding how genomic implementation affects a health system all the way from patients to hospital leadership requires further understanding. To address this gap, we conducted a formal qualitative study, using the Consolidated Framework for Implementation Research (CFIR), ${ }^{25}$ to identify system-level factors that have played a role in the implementation of genomic medicine applications represented by the IGNITE network studies.

\section{MATERIALS AND METHODS}

\section{Participants}

We interviewed study team members from each of six IGNITE projects, including a principal investigator (PI) and the project manager from each project. We asked the PI of each project to name two additional individuals from their project who could address barriers and facilitators to implementation. These individuals were typically coordinators or a study co-investigator from one of their IGNITE project sites.

\section{Instrument development and recruitment}

The study team developed a semistructured interview guide (see Appendix A), based upon the Consolidated Framework for Implementation Research (CFIR). ${ }^{25}$ The CFIR is one approach to understanding the success or failure of integration of any aspect of genomic medicine into clinical practice, and includes multiple constructs that allow one to analyze a variety of applications represented by the IGNITE network, including those that may be multifactorial and representative of the patient, provider, organization, payer, and policy levels. ${ }^{25}$ While CFIR includes five domains-inner setting, outer setting, characteristics of the people involved, the intervention characteristics, and the implementation process - we chose to focus specifically on the domain of the inner setting based upon its relevance to our initial research question, which was to identify system-level factors affecting implementation of genomic research findings into the clinical setting. The broad range of practice settings in the IGNITE project provided important input that can be useful across different organizations (e.g., academic and nonacademic) and different practice settings (e.g., specialty care and general practice, community-based and urban-based).

A study coordinator sent an email describing the study to potential interviewees and scheduled a telephone interview, regardless of if projects were in the initiation or recruitment phase. The interviews followed the semistructured interview guide (see Appendix A), which included questions asking interviewees to describe their study site(s), the progress at each site, and factors facilitating or impeding implementation of the project. Refinement of the interview guide was an iterative process: after the first three interviews, the questions were revised for clarity. Interviews were conducted by two of the investigators (B.A.B. and N.R.S.). We probed on responses to specifically ask about several organizational factors, including (1) infrastructure changes needed to accommodate the intervention, (2) stakeholder engagement, (3) adequacy of resources to support the implementation, (4) level of 
Table 1 Barriers and facilitators to genomic testing implementation based on the CFIR framework inner setting constructs ${ }^{25}$ CFIR framework: inner setting

\begin{tabular}{|c|c|c|}
\hline Applied construct & CFIR construct & Implementation application for research and clinical practice \\
\hline $\begin{array}{l}\text { Incorporating genomics into electronic } \\
\text { health records }\end{array}$ & $\begin{array}{l}\text { Available Resources Stakeholder } \\
\text { Engagement }\end{array}$ & $\begin{array}{l}\text { - Lack of integration of decision support tools or testing results into } \\
\text { electronic health records (EHR) } \\
\text { - Current technology lacks appropriate computer logic for interpretation of } \\
\text { genomic testing } \\
\text { - Required IT collaboration for integration }\end{array}$ \\
\hline Reimbursement & Infrastructure Changes & $\begin{array}{l}\text { - Lack of funding for testing outside of research setting } \\
\text { - Limited avenues for reimbursement for genomic testing in current health- } \\
\text { care climate }\end{array}$ \\
\hline $\begin{array}{l}\text { Clinician champions as facilitators of } \\
\text { genomic implementation }\end{array}$ & $\begin{array}{l}\text { Stakeholder Engagement } \\
\text { Leadership Engagement }\end{array}$ & $\begin{array}{l}\text { - Establishment of physician leadership on a departmental level to assist } \\
\text { with recruitment and buy-in } \\
\text { - Use of early adopters to encourage participation from other clinicians } \\
\text { - Recommendation to use testing and support tools between clinicians }\end{array}$ \\
\hline
\end{tabular}

CFIR Consolidated Framework for Implementation Research, IT information technology.

organizational receptivity to the project, and (5) perceived need for their intervention.

All interviews were digitally recorded over the phone after obtaining verbal consent. The interviews lasted between 15 and 70 minutes, and were transcribed, de-identified and entered into QSR International's NVivo 10.0 for coding and analysis.

\section{Data analysis}

Study investigators met after reviewing a subset of transcripts to develop a coding system through an iterative process standard for content analysis. ${ }^{26}$ Initial codes related directly to the CFIR framework inner setting as well as questions asked during the interviews (for example, barriers to implementation, progress of the project at various sites, etc.). Subsequent codes were added that emerged through a close reading of the data. Three transcripts were coded by two investigators during the initial codebook development phase, with an additional eight transcripts independently coded by these investigators. Once the codebook was stabilized, all transcripts were double coded. Differences in coding were resolved by consensus to achieve $100 \%$ consistency. After all transcripts were coded, the investigators reviewed the coded data to identify dominant themes. ${ }^{26}$ We selected representative quotes to illustrate pertinent findings. The study protocol was classified as exempt by the Institutional Review Board of the University of Pennsylvania.

\section{RESULTS}

Twenty persons associated with the IGNITE network participated in the open-ended semistructured interviews. These included six principal investigators, five project 
managers, and nine study coordinators. No network members refused participation.

Guided by the CFIR constructs, system- and site-level barriers and facilitators to implementation of genomic medicine research and interventions, as well as clinical factors, were identified. Overall, a majority of those interviewed discussed patient and site characteristics including demographics, geographic location, size of clinic or hospital, and clinic setting (rural/urban, academic/community). These characteristics were often presented as both barriers and facilitators based on the stage of the project and whether studies were designed to recruit patients for genomic testing or clinicians for changes in practice. Stage of project implementation also varied among IGNITE project sites, with some interviews including discussion of implementation of the research project, while others focused on the implementation of genomic testing in clinical care. The CFIR innersetting constructs identified across IGNITE projects, with examples for both research and overall genomic testing implementation, are presented below. Table 1 summarizes the study findings.

\section{Barriers}

Incorporating genomics into electronic health records Limitations in technology, specifically integration of new genomic data and clinical decision support tools into electronic health records (EHR), were identified as barriers to implementation for both research and clinical genomic testing. Integration of test results and incorporation of clinical decision support tools were goals when considering changes to the EHR. Limitations within current medical record systems were mentioned in a number of interviews, and common concerns included the inability to automatically upload data elements, having to scan results into the EHR, and as one principal investigator discussed:

Their electronic health record actually does not have...the... functionality that allows them to do interruptive clinical decision support... (PI)

Interviewees also mentioned that making technological advancements within hospitals and clinics was crucial for implementing genetic and genomic testing into everyday practice. As one principal investigator pointed out:

...incorporation of computer logic at the point of care is going to be the only way that genomics can work. And consequently-right now, if you look at the major vendors... they don't have a lot in the way of genomics incorporated. (PI)

This PI stressed the fact that implementation of genomic testing would require EHR vendors to update their systems and incorporate the necessary technologies to facilitate testing.

\section{Reluctance to participate in genomics studies}

Participants described a culture of physician reluctance to participate in genomic focused clinical studies due to unfamiliarity with genomic testing or ambiguity in the interpretation and application of its results. This reluctance stemmed from clinician difficulty in envisioning how implementation of genetic testing would improve patient health or a low comfort level in discussing results if implications were unknown. One principal investigator mentioned the lack of buy-in from clinicians without a guarantee of benefit for patients:

...there wasn't real research buy-in. I think this is a research naïve site. And they said, well, is it definitely going to help our patients? And we said no, because with research you never know if it will definitely help your patients. And they said, well, if it's not definitely going to help our patients, we're not going to do it. (PI)

Along with physician concerns, patient support was also perceived to be lacking, which negatively affected the study recruitment process. Difficulties with both patient and physician recruitment were expressed by a principal investigator who mentioned how the idea of doing research studies in a clinical setting resulted in reduced participation:

The biggest thing I think is often the barrier can be that you say the word "research"-and the community engagement person said this, too-and you can scare people off. And so, getting people to understand that this is research in the enhancement of clinical care. (PI)

Participants linked physician and patient concerns about being involved in clinical studies with difficulty in implementing genomic testing in practice.

\section{Uncertain reimbursement for clinical programs}

Some interviewees felt that the testing for genomic markers could not be implemented into clinical practice due to the uncertainty of reimbursement outside of a research study. One principal investigator noted that, although hospital administration was supportive of research, obstacles in a non-research setting would be higher due to the cost of the test:

The big thing is the reimbursement. I mean, we approached them and they were extraordinarily receptive and helpful. But we made it clear that this was a research study and that the cost would not be relayed to the patient, would not be relayed to the health system.... I think the obstacles in a nonresearch setting would be much higher if these are costly tests and not being reimbursed by the payers. (PI)

\section{Facilitators}

\section{Investigator and clinician communication}

The majority of those interviewed discussed that good communication among sites of an IGNITE project and within individual projects were facilitators to implementation of the studies. With respect to the former, various forms of communication, including webinars, conference calls, and presentations, were used to solicit feedback and unify study sites. Collaborative strategies were helpful for the majority of those who mentioned them, with one principal investigator commenting:

It's just a reminder of how great teamwork is when it works, because I couldn't have any idea. What can you do in EPIC 
and what can you do with the interface? And then you have all these different people who know. (PI)

This same principal investigator also mentioned how monthly meetings among sites within an IGNITE project allowed for the opportunity to discuss problems and provide feedback:

Yeah, very helpful. We basically-the format is basically we start with the progress of the study, and then we bring up just different kinds of issues that affect all of us...But it's just kind of nice to have one meeting a month where people can just sort of talk about things that are concerning. (PI)

Communication among projects was necessary for successful implementation of each research study and for consistency in implementation, but internal communication within a given site was noted as being critical to continued success both throughout the study period and during the transition from research into clinical practice. Interviewees described that regular feedback from clinicians about genomic testing integration into current practice was important. One study coordinator noted that feedback allowed staff to modify their approach, if necessary, to get physicians involved or patients recruited into research studies. One principal investigator stressed the importance of communication among stakeholders as essential for long-term success:

And they have so much to do and so many different things to deal with that they just maybe don't want to think about something that's new and seems challenging.... And so I think what is going to be interesting over probably the next year as that program matures is that-my guess is that that's going to rub off-right? He's going to talk to his colleagues and say this is really helpful.... But I think we can already project that as physicians feel like it's helpful in clinical practice, they're going to share that with their colleagues. (PI)

A project manager also mentioned that physician feedback allowed for better gauging of overall interest and engagement in the project:

We have a lot of clinicians here that are engaged in the project. So we're getting feedback about that. So it shows me that there is an interest and curiosity at trying to get more engaged in the protocol and understand that. (Project Manager)

\section{Clinical leadership as facilitators of genomic implementation}

Similar to internal communication, clinician enthusiasm, support, and championing genomic testing throughout the study period and into clinical practice was mentioned by all sites as a facilitator for implementation. This included backing by physician leaders and early adopters. Without this encouragement, studies at individual sites would find it extremely challenging to implement genomic testing. One principal investigator discussed physicians who were early adopters and the benefit of early interest in moving the study forward:

...trying to figure out where people are on the spectrumwhere the clinicians are on that spectrum, I think is important to helping move them along. Because if their inclination is to be generally an early adopter or for whatever reason they're interested specifically in this and willing to be an early adopter, then it's just an easier conversation than if somebody views this as just another thing that's going to complicate their practice and take more time that they don't have. (PI)

Hospital leadership engagement throughout programming Along with physician guidance facilitating the implementation, many interviewees also mentioned high-level hospital leadership engagement as important to study initiation and long-term success. The same principal investigator who discussed the importance of physicians also commented on how leadership made their project a priority:

I think we had high-level support in the right places.... So I think the fact that our chief information officer totally bought into this, and basically-especially early on pushed some of our things to the front of the line, made them a high priority with her team. I think that that really helped. (PI)

Another principal investigator commented on how engagement from hospital leadership helped with funding:

[the site] took on our-the IT aim...because the Chief Medical Officer and the Chief Information Officer both knew about [the program] and were incredibly supportive about what it was doing. So they decided that they would take on the integration with the IT system themselves without any additional funding. (PI)

The general consensus was that the long-term success of genomic testing in the clinical realm required the support of hospital leadership, with one project manager echoing this view:

Basically, I think it comes down to a lot of discussions at the institutional level to get the concept approved, where you're talking to the CMO or the Chief Medical Officer in the hospital to prove that or to show that there is value.... And then from there it just helps grease the wheels in actually making it happen with some of the clinics. (Project Manager)

\section{DISCUSSION}

Until now, there had been limited examination of the barriers and facilitators to implementation of genomic medicine in clinical practice, particularly from an organizational and system-level perspective. ${ }^{8,27,28}$ While Manolio et al., provided a summary of potential challenges to genomic implementation, ${ }^{8}$ this study, as a next step, now directly addresses some of these potential challenges by conducting qualitative interviews, guided by the CFIR framework, ${ }^{25}$ with research professionals on a wide range of clinical topics. Several CFIR inner-setting constructs emerged as being consistent and important across IGNITE projects, with distinct differences between facilitators and barriers to implementation. Slow technological advancement and EHR integration, skepticism and reluctance toward genetic medicine from physicians, as well as slow recruitment of patients, were barriers to implementation mentioned across IGNITE projects. Our findings build on prior work by Gottesman et al., that 
showed how EHRs can help facilitate the implementation of genomic medicine into practice, emphasizing the need for more rapid EHR integration. ${ }^{29}$ This initial integration of genomic medicine into EHRs may provide a path to easier implementation of testing by physicians. Additionally, lack of reimbursement for genetic medicine was mentioned as a major barrier to implementation in a clinical setting with both physicians and leadership voicing concern about reimbursement from insurance companies.

Communication among a variety of stakeholders within and between IGNITE projects, including feedback on successes and failures, were important facilitators and keys to success. This communication and feedback was seen as critical to engagement and buy-in by physicians and hospital management, and particularly useful for both implementation of initial genomic research studies and eventually for clinicians to incorporate genetic medicine into usual care. A lack of physician buy-in has also been shown in other studies to be a barrier to implementation, and overcoming this barrier is an eventual necessity to achieve successful implementation. ${ }^{10,11,30}$ In a study by Hamilton et al., physicians did not view genetic services as being advantageous or necessary for patient care; however, those physicians who were already versed and interested in genetic medicine were also more likely to implement it. ${ }^{30}$ Similar findings were echoed throughout the IGNITE study with interviewees expressing that clinicians seemed uncertain as to how genetic medicine would help their patients or provide benefit beyond current practice standards.

Finally, patient recruitment into genomic testing studies, as a first step toward demonstrating the utility of genetic testing in practice, was shown to be an important barrier to ultimate implementation. Access to patients, as well as logistical challenges of working within the constraints of clinic visits, was challenging at many of the IGNITE sites. This further explores the importance of incorporating patients into the testing process as described by the IGNITE network. ${ }^{12}$ These difficulties with patient recruitment, together with the fact that physicians want evidence before they implement a new intervention, highlights the importance of identifying solutions to improving clinical study implementation.

While barriers such as technological advancements and reimbursement may not be readily solved, physician buy-in may be addressed with increased information about genomic testing early in medical training. Our findings suggest that this education may then benefit from continued open communication and feedback between researchers, clinicians, and administrators. Nonetheless, even with increased education, technological and financial barriers will remain important obstacles to implementing genomics in practice. These needs, along with increasing patient participation in genetic and genomic testing, require further exploration.

There were several limitations in this study. First, only research faculty and staff involved with the IGNITE projects were interviewed. Thus, no clinicians, staff, or hospital administration perspectives were provided on implementation, although they communicated their viewpoints with interviewees. Getting information indirectly from these individuals may result in differences in opinions expressed about implementation between those interviewed and those who work on the front lines in the clinics. This may be particularly important given that many of the views shared by interviewees describe feelings about stakeholder participation or reluctance, and reasons for these may differ if clinicians, patients, or hospital leadership were interviewed themselves. Additionally, many of the interviews were conducted while project sites were still in the project initiation or recruitment phase, which may have limited the topics of conversation to recruitment challenges for research instead of discussion about implementation of genomic medicine on a broader scale. While these early phases of implementation are important to understand, interviews at later stages of project implementation may garner different information. Finally, a widespread group of sites within each of the IGNITE projects were discussed by interviewees. While this allowed for a robust discussion of different challenges and best practices, the barriers may be specific to both research and to an individual site.

Overall, this study provides important information about barriers and facilitators to studying and implementing genomic medicine. Efforts in the future might be directed toward incorporating genetic information and results into hospital EHR, as well as research to determine if reimbursement is warranted for genomic medicine or alternative methods for incorporation of testing if reimbursement is not feasible. It may be crucial to address these challenges before widespread implementation can be accomplished. Future research in the area of genomic medicine implementation can expand on these findings by incorporating the perspective of additional stakeholders including patients, administrators, and clinicians.

\section{ELECTRONIC SUPPLEMENTARY MATERIAL}

The online version of this article (https://doi.org/10.1038/s41436018-0378-9) contains supplementary material, which is available to authorized users.

\section{ACKNOWLEDGEMENTS}

We thank Selena Suhail-Sindhu, recent graduate of the public health program at Columbia University, for her assistance with data collection and management, and Kara Silberthau, medical student at the University of Pennsylvania, for her contribution to data management and analysis. Support for this study was provided from the Implementation Science Working Group at the University of Pennsylvania and the NHGRI U01-HG007266. Additional IGNITE network grants include U01-HG007269, U01HG007253, U01-HG007762, U01-HG007282, U01-HG007775, and U01-HG007278.

\section{DISCLOSURE}

The authors declare no conflicts of interest. 


\section{REFERENCES}

1. Collins FS, McKusick VA. Implications of the Human Genome Project for medical science. JAMA. 2001;285:540-544.

2. Subramanian G, Adams MD, Venter JC, Broder S. Implications of the human genome for understanding human biology and medicine. JAMA. 2001;286:2296-2307.

3. Collins FS, Varmus $\mathrm{H}$. A new initiative on precision medicine. $\mathrm{N}$ Engl J Med. 2015:372:793-795.

4. McCarthy JJ, McLeod HL, Ginsburg GS. Genomic medicine: a decade of successes, challenges, and opportunities. Sci Transl Med. 2013;5:189sr184.

5. Green ED, Guyer MS. National Human Genome Research I. Charting a course for genomic medicine from base pairs to bedside. Nature. 2011;470:204-213.

6. Ginsburg GS, Willard HF. Genomic and personalized medicine: foundations and applications. Transl Res. 2009;154:277-287.

7. Relling MV, Evans WE. Pharmacogenomics in the clinic. Nature 2015;526:343-350.

8. Manolio TA, Chisholm RL, Ozenberger B, et al. Implementing genomic medicine in the clinic: the future is here. Genet Med. 2013;15: 258-267.

9. Hofman V, llie M, Long $E$, et al. Detection of circulating tumor cells from lung cancer patients in the era of targeted therapy: promises, drawbacks and pitfalls. Curr Mol Med. 2014;14:440-456.

10. Burke W, Korngiebel DM. Closing the gap between knowledge and clinical application: challenges for genomic translation. PLoS Genet. 2015;11:e1004978.

11. Williams MS. Genomic medicine implementation: learning by example. Am J Med Genet C Semin Med Genet. 2014;166C:8-14.

12. Sperber NR, Carpenter JS, Cavallari LH, et al. Challenges and strategies for implementing genomic services in diverse settings: experiences from the Implementing GeNomics In pracTicE (IGNITE) network. BMC Med Genom. 2017;10:35

13. Grosse SD, McBride CM, Evans JP, Khoury MJ. Personal utility and genomic information: look before you leap. Genet Med. 2009:11:575-576.

14. Bunnik EM, Janssens AC, Schermer MH. Personal utility in genomic testing: is there such a thing? J Med Ethics. 2015;41:322-326.

15. Botkin JR, Teutsch SM, Kaye $\mathrm{Cl}$, et al. Outcomes of interest in evidencebased evaluations of genetic tests. Genet Med. 2010;12:228-235.

16. Hofman KJ, Tambor ES, Chase GA, Geller G, Faden RR, Holtzman NA. Physicians' knowledge of genetics and genetic tests. Acad Med. 1993:68:625-632.
17. Hayflick SJ, Eiff MP. Role of primary care providers in the delivery of genetics services. Community Genet. 1998;1:18-22.

18. Suther S, Goodson P. Barriers to the provision of genetic services by primary care physicians: a systematic review of the literature. Genet Med. 2003; 5:70-76

19. Weipert CM, Ryan KA, Everett JN, et al. Physician experiences and understanding of genomic sequencing in oncology. J Genet Couns. 2018:27:187-196. https://doi.org/10.1007/s10897-017-0134-3.

20. Vassy JL, Green RC, Lehmann LS. Genomic medicine in primary care: barriers and assets. Postgrad Med J. 2013;89:615-616.

21. Mikat-Stevens NA, Larson IA, Tarini BA. Primary-care providers' perceived barriers to integration of genetics services: a systematic review of the literature. Genet Med. 2015;17:169-176.

22. Chow-White P, Ha D, Laskin J. Knowledge, attitudes, and values among physicians working with clinical genomics: a survey of medical oncologists. Hum Resour Health. 2017;15:42.

23. Hazin R, Brothers KB, Malin BA, et al. Ethical, legal, and social implications of incorporating genomic information into electronic health records. Genet Med. 2013:15:810-816.

24. Weitzel KW, Alexander M, Bernhardt BA, et al. The IGNITE network: a model for genomic medicine implementation and research. BMC Med Genom. 2016;9:1

25. Damschroder LJ, Aron DC, Keith RE, Kirsh SR, Alexander JA, Lowery JC. Fostering implementation of health services research findings into practice: a consolidated framework for advancing implementation science. Implement Sci. 2009;4:50.

26. Huberman AM, Miles MB. Understanding and validity in qualitative research. In: Huberman AM, Miles MBThe qualitative researcher's companion. Thousand Oaks, CA: SAGE; 2002. p. 36-65.

27. Luzum JA, Pakyz RE, Elsey $A R$, et al. The Pharmacogenomics Research Network Translational Pharmacogenetics Program: outcomes and metrics of pharmacogenetic implementations across diverse healthcare systems. Clin Pharmacol Ther. 2017;102:502-510.

28. Shuldiner AR, Relling MV, Peterson JF, et al. The Pharmacogenomics Research Network Translational Pharmacogenetics Program: overcoming challenges of real-world implementation. Clin Pharmacol Ther 2013:94:207-210.

29. Gottesman O, Kuivaniemi H, Tromp G, et al. The Electronic Medical Records and Genomics (eMERGE) Network: past, present, and future. Genet Med. 2013;15:761-771.

30. Hamilton AB, Oishi S, Yano EM, Gammage CE, Marshall NJ, Scheuner MT. Factors influencing organizational adoption and implementation of clinical genetic services. Genet Med. 2014:16:238-245. 